Research article Open Access

\title{
Cartilage markers and their association with cartilage loss on magnetic resonance imaging in knee osteoarthritis: the Boston Osteoarthritis Knee Study
}

\author{
David J Hunter ${ }^{1}$, Jiang Li', Michael LaValley¹, Doug C Bauer², Michael Nevitt², Jeroen DeGroot ${ }^{3}$, \\ Robin Poole ${ }^{4}$, David Eyre ${ }^{5}$, Ali Guermazi ${ }^{1}$, Dan Gale ${ }^{6}$ and David T Felson${ }^{1}$
}

\author{
${ }^{1}$ Clinical Epidemiology Research and Training Unit, Boston University School of Medicine, Boston, MA, USA \\ 2University of California, San Francisco, San Francisco, CA 94107, USA \\ 3Immunological and Infectious Diseases Division, TNO Quality of Life, Leiden, The Netherlands \\ ${ }^{4}$ McGill University, Montreal, QC, Canada \\ ${ }^{5}$ Department of Orthopaedics and Sports Medicine, University of Washington, Seattle, WA 98195, USA \\ ${ }^{6}$ VirtualScopics, Rochester, NY 14625, USA
}

Corresponding author: David J Hunter, djhunter@bu.edu

Received: 4 Apr 2007 Revisions requested: 23 May 2007 Revisions received: 12 Sep 2007 Accepted: 24 Oct 2007 Published: 24 Oct 2007

Arthritis Research \& Therapy 2007, 9:R108 (doi:10.1186/ar2314)

This article is online at: http://arthritis-research.com/content/9/5/R108

(c) 2007 Hunter et al.; licensee BioMed Central Ltd.

This is an open access article distributed under the terms of the Creative Commons Attribution License (http://creativecommons.org/licenses/by/2.0), which permits unrestricted use, distribution, and reproduction in any medium, provided the original work is properly cited.

\begin{abstract}
We used data from a longitudinal observation study to determine whether markers of cartilage turnover could serve as predictors of cartilage loss on magnetic resonance imaging (MRI). We conducted a study of data from the Boston Osteoarthritis of the Knee Study (BOKS), a completed natural history study of knee osteoarthritis (OA). All subjects in the study met American College of Rheumatology criteria for knee $\mathrm{OA}$. Baseline and follow-up knee magnetic resonance images were scored for cartilage loss by means of the WORMS (Whole Organ Magnetic Resonance Imaging Score) semiquantitative grading scheme. Within the BOKS population, 80 subjects who experienced cartilage loss and 80 subjects who did not were selected for the purposes of this nested case control study. We assessed the baseline levels of cartilage degradation and synthesis products by means of assays for type I and II cleavage by collagenases (Col2:3/4 $\mathrm{C}_{\text {short }}$ or $\mathrm{C} 1,2 \mathrm{C}$ ), type II cleavage only with Col2:3/4 $\mathrm{C}_{\text {longmono }}(\mathrm{C} 2 \mathrm{C})$, type II synthesis (C-propeptide), the C-telopeptide of type II (Col2CTx), aggrecan 846 epitope, and cartilage oligomeric matrix protein (COMP). We performed a logistic regression to examine the relation of levels of each biomarker to the risk of cartilage loss in any knee. All analyses

were adjusted for gender, age, and body mass index (BMI); results stratified by gender gave similar results. One hundred thirty-seven patients with symptomatic knee OA were assessed. At baseline, the mean (standard deviation) age was 67 (9) years and $54 \%$ were male. Seventy-six percent of the subjects had radiographic tibiofemoral OA (Kellgren \& Lawrence grade of greater than or equal to 2) and the remainder had patellofemoral OA. With the exception of COMP, none of the other biomarkers was a statistically significant predictor of cartilage loss. For a 1unit increase in COMP, the odds of cartilage loss increased 6.09 times (95\% confidence interval [Cl] 1.34 to 27.67). After the analysis of COMP was adjusted for age, gender, and BMI, the risk for cartilage loss was 6.35 (95\% Cl 1.36 to 29.65). Among subjects with symptomatic knee OA, a single measurement of increased COMP predicted subsequent cartilage loss on MRI. The other biochemical markers of cartilage synthesis and degradation do not facilitate prediction of cartilage loss. With the exception of COMP, if changes in cartilage turnover in patients with symptomatic knee OA are associated with cartilage loss, they do not appear to affect systemic biomarker levels.
\end{abstract}

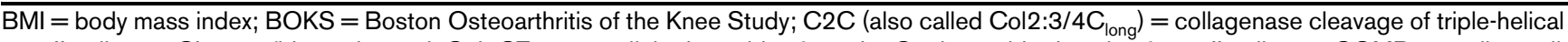
type II collagen; $\mathrm{Cl}=$ confidence interval; Col2 $\mathrm{CTx}=$ crosslinked peptides from the C-telopeptide domain of type II collagen; COMP = cartilage oligomeric matrix protein; CPII = C-propeptide of type II collagen; ELISA = enzyme-linked immunosorbent assay; FOV $=$ field of view; $K \& L=K e l l g r e n$ \& Lawrence; $\mathrm{MRI}=$ magnetic resonance imaging; $\mathrm{OA}=$ osteoarthritis; TE = time to echo; TR = repetition time; WORMS = Whole Organ Magnetic Resonance Imaging Score. 


\section{Introduction}

Osteoarthritis (OA) is characterized by the degeneration of articular cartilage. This results from a direct attack on matrix molecules, resulting in their cleavage, damage to these molecules, and their loss. It is also accompanied by a response of the tissue to this damage which involves enhanced matrix synthesis and turnover. The most direct evidence of pathology is cartilage degradation. A secondary and more indirect indication is cartilage matrix synthesis. The amount of synthesis in relation to degradation may prove of great importance in determining disease progression [1].

The ability to use biochemical markers to predict disease progression and identify patients most likely to progress is a top priority in the future management of OA. Ultimately, it would enable much more rapid assessment of structure-modifying therapies in clinical trials. It may also allow the identification of patients at highest risk of progression, allowing the efficient testing of new treatments. Biochemical markers of OA progression represent a surrogate for structural change which may have advantages over existing methods of measuring structure. Therapeutic development in $\mathrm{OA}$ is constrained by the slow progress of structural changes using standard imaging techniques. The development and validation of biochemical markers may accelerate the pace of therapeutic development.

Some recent work on type II collagen has suggested that assays for type II collagen degradation, when used in combination or with markers of collagen synthesis, can distinguish populations with knee OA which exhibit progression of joint damage from non-progressors. The ratio of the type II collagen crosslinking C-telopeptide (CTX-II) to the amino propeptide of type IIA collagen [2] or the ratio of two collagenase-generated cleavage epitopes in the helical region $(\mathrm{C} 1,2 \mathrm{C}$ to $\mathrm{C} 2 \mathrm{C})$ [3] each can make this distinction. The results from each of these studies need to be confirmed. But, clearly, these two independent studies point to differences in collagen turnover as being suggestive of disease progression and provide encouragement for future work in this area. Preliminary plain radiographic studies suggest that cartilage oligomeric matrix protein (COMP) may be a useful prognostic marker of disease progression in knee [4-6] and hip [7] OA.

The overarching aim of this investigation was to conduct a study within an existing longitudinal dataset of knee OA with serial knee magnetic resonance imaging (MRI) to evaluate and validate promising biochemical markers, markers that have been reported in either cross-sectional or longitudinal studies to be related to OA or its progression. MRI of the knee has the advantage of covering the whole joint in one examination, meaning that the cartilage defects in the joint can be visualized directly, regardless of their location [8]. Direct visualization of cartilage defects enhances the ability to detect cartilage loss that can be missed using joint space narrowing from plain radiographs $[8,9]$.

More specifically, we assessed the baseline levels of cartilage degradation, synthesis, and turnover products using collagenase-generated $\mathrm{C} 1,2 \mathrm{C}$, and $\mathrm{C} 2 \mathrm{C}$; Col II C-telopeptide (CTX-Il assay); C-propeptide of type II collagen; aggrecan 846 epitope; and COMP in a sample of knees with known knee cartilage loss and controls. Our prior hypotheses were that increased levels of cartilage degradation products would be predictive of cartilage loss and that imbalance of cartilage synthesis and degradation would be predictive of cartilage loss.

\section{Materials and methods Study sample}

We conducted an analysis of data from the Boston Osteoarthritis of the Knee Study (BOKS), a completed natural history study of knee OA [10]. To be eligible for the study, patients had to have knee pain, aching, or stiffness on most days within the last month and they had to have reported that a physician had told them that they had arthritis in the knee. If they met both of those criteria, they underwent radiography (weightbearing fluoroscopic posteroanterior, lateral, and skyline views) and if on any of these views they had a definite osteophyte in the symptomatic knee (either tibiofemoral or patellofemoral), they were eligible for the study. In addition, they had to fill out a questionnaire that screened for other forms of arthritis, including rheumatoid arthritis, and information on the use of medications for arthritis was gathered. If a patient screened positive for another form of arthritis or had been receiving medications that were appropriate for rheumatoid arthritis or other forms of arthritis, he or she was excluded. Thus, all subjects in the study had primary clinical knee OA and met American College of Rheumatology criteria for this disorder.

Of 324 subjects who entered the study, $86 \%$ completed a full comprehensive follow-up at a later time point (15 and/or 30 months). These comprehensive examinations consisted of an MRI of the more affected knee and a comprehensive set of radiographs, including a semiflexed fluoroscopically positioned posteroanterior radiograph using the method of Chaisson and colleagues [11] and Buckland-Wright [12].

Blood and urine (second morning void) specimens were also obtained at baseline. Specimens were aliquoted and immediately frozen; serum was frozen at $-70^{\circ} \mathrm{C}$ and urine at $-20^{\circ} \mathrm{C}$. The specimens were stored at a long-term repository (the Biomedical Research Institute, Rockville, MD, USA).

Within the BOKS population, 80 subjects with MRI cartilage loss and 80 subjects without cartilage loss were selected for the purposes of this nested case control study. Cartilage loss was defined as an increase in cartilage score at 30 months from that at baseline. After completion of the assays, 153 par- 
ticipants had data available for all of the biomarker assays. Once the biomarker assay data and MRI data were merged, 137 subjects had complete data (both complete biomarker and longitudinal MRI data) available for analysis. These participants were similar to those from the larger study sample. The institutional review boards of Boston University Medical Center and the Veterans Administration Boston Health Care System approved the baseline and follow-up examinations, and informed consent was obtained from all participants.

\section{Magnetic resonance imaging}

All studies were performed with a Signa 1.5T MRI system (General Electric Comp., Milwaukee, WI, USA) using a phased-array knee coil. A positioning device was used to ensure uniformity of positioning among patients. The imaging protocol included sagittal spin-echo proton density- and T2weighted images (repetition time [TR] 2,200 milliseconds and time to echo [TE] 20/80 milliseconds) with a slice thickness of $3 \mathrm{~mm}$, a 1-mm interslice gap, 1 excitation, a field of view (FOV) of 11 to $12 \mathrm{~cm}$, and a matrix of $256 \times 192$ pixels and coronal and axial spin-echo fat-suppressed proton density- and T2weighted images (TR 2,200 milliseconds and TE 20/80 milliseconds) with a slice thickness of $3 \mathrm{~mm}$, a $1-\mathrm{mm}$ interslice gap, 1 excitation, and the same FOV and matrix.

Cartilage on MRI was scored paired and unblinded to sequence on 14 plates (anterior, central, and posterior femur; anterior, central, and posterior tibia; and medial and lateral patella) using the Whole Organ Magnetic Resonance Imaging Score (WORMS) semiquantitative method [13]. Both cartilage signal and morphology were scored using a 0-to-6 scale: $0=$ normal thickness and signal, $1=$ normal thickness but increased signal on T2-weighted images, 2 = solitary focal defect of less than $1 \mathrm{~cm}$ in greatest width, $3=$ areas of partialthickness defects (less than $75 \%$ of the plate) with areas of preserved thickness, $4=$ diffuse partial-thickness loss of cartilage (greater than or equal to $75 \%$ of the plate), $5=$ areas of full-thickness loss (less than $75 \%$ of the plate) with areas of partial-thickness loss, and $6=$ diffuse full-thickness loss (greater than or equal to $75 \%$ of the plate). The intraclass correlation coefficient on agreement for cartilage readings ranged from 0.75 to 0.97 .

In WORMS, grade 1 does not represent a morphologic abnormality but rather a change in signal in cartilage of otherwisenormal morphology. Grades 2 and 3 represent similar types of abnormality of the cartilage, focal defects without overall thinning. Therefore, to create a consistent and logical scale for evaluation of cartilage morphologic change, we collapsed the WORMS cartilage score to a 0-to-4 scale in which the original WORMS score of 0 and 1 were collapsed to 0 , the original scores of 2 and 3 were collapsed to 1 , and the original scores of 4,5 , and 6 were considered 2,3 , and 4 , respectively. Cartilage loss was defined as an increase in the score at any sub- region compared to baseline in any of the 14 subregions of the knee scored for cartilage in each knee.

We selected subjects who attended the baseline and final visits with an intervisit duration generally more than $\mathbf{3 0}$ months. Within the BOKS population, all of the biomarkers mentioned and cartilage loss on serial MRI were available on 137 participants.

\section{Cartilage biomarkers}

The neoepitope resulting from collagenase cleavage of triplehelical type II collagen (Col2:3/4 $\mathrm{C}_{\text {long, }}$, also known as $\mathrm{C} 2 \mathrm{C}$ ) was measured by means of an enzyme-linked immunosorbent assay (ELISA) [14]. It uses a monoclonal antibody that recognizes a sequence near the carboxy terminus of the $3 / 4$ piece.

The $\mathrm{C} 1,2 \mathrm{C}$ assay relates to epitopes formed by degradation of type II collagen by collagenase 1, 2, and 3 [15]. Serum concentrations of these degradation products is determined by inhibition ELISA using polyclonal rabbit anti-human antibody.

The C-propeptide of type II collagen (CPII) is cleaved from the procollagen molecule as it forms fibrils. Thus, CPII levels are potentially an index of collagen type II formation and are measured with an ELISA [16].

Aggrecan 846 epitope is present on intact aggrecan molecules (the epitope is associated with chondroitin sulfate chains near the G3 domain) [17]. Aggrecan 846 is measured by ELISA with a mouse monoclonal immunoglobulin $M$ antibody [18].

The C2C, C1,2C, C-propeptide (CPII), and CS 846 commercial assays were obtained from IBEX Technologies Inc. (Montreal, QC, Canada). These have been validated for human studies $[19,20]$.

The intraday $(n=20)$ and interday $(n=200)$ coefficients of variance for each biomarker are, respectively, $10 \%-17 \%$ and $14 \%$ for $\mathrm{C} 2 \mathrm{C}, 5 \%-14 \%$ and $13 \%$ for $\mathrm{C} 1,2 \mathrm{C}, 4 \%-12 \%$ and $12 \%$ for CS 846 epitope, and $11 \%-18 \%$ and $16 \%$ for CPII. The interassay coefficients of variance for all the assays are in the range of $6.4 \%$ to $11.5 \%$ [19].

Crosslinked peptides from the C-telopeptide domain of type II collagen (Col2CTx) were quantified by competition ELISA. The assay is based upon a monoclonal antibody, 2B4, which was raised in mice against a conjugated synthetic peptide, EKGPDP [21]. This assay was conducted in the laboratory of author DE. The Col2CTx ratio was Col2CTx/urinary creatinine. The intra-assay and interassay coefficients of variation for the CTx-II/Cr assay were $6 \%$ and $13 \%$, respectively [22].

COMP was measured in serum [23] by a solid-phase two-site enzyme immunoassay. It is based on the direct sandwich tech- 
nique, in which two monoclonal antibodies are directed against separate antigenic determinants on the COMP molecule (intra-assay and interassay variations of less than $5 \%$ and a detection limit of less than $0.1 \mathrm{U} / \mathrm{L}$ ). This is a commercial assay manufactured by AnaMar Medical R\&D (Lund, Sweden). With the exception of Col2CTX, all assays were conducted at TNO (Leiden, The Netherlands).

\section{Statistical analysis}

Hypothesis 1: increased levels of cartilage degradation products are predictive of cartilage loss

We assessed whether increased levels of each biomarker were predictive of subsequent cartilage loss on knee MRI (ascertained from baseline to visit at $\mathbf{3 0}$ months). The six biochemical markers used as predictor variables were Col2:3/ $4 \mathrm{C}_{\text {long }}$; $\mathrm{C} 1,2 \mathrm{C}$; Col II C-telopeptide; C-propeptide of type II collagen; aggrecan 846 epitope; and COMP. We used the standardized variable as a predictor to facilitate comparison between the multiple biomarkers.

We firstly assessed the distribution of baseline cartilage scores to ensure that all participants were capable of continued cartilage loss and had not reached a ceiling. We then performed a logistic regression to examine the relation of levels of each logarithmic transformed biomarker to the risk of cartilage loss in any plate. Cartilage loss in a knee was defined as an increase in cartilage score in any of the 14 subregions scored for cartilage in each knee. Considering that the risk profiles of cartilage loss and magnitude of effect of a particular biomarker on cartilage loss may be different between men and women, we first conducted separate analyses for each gender. As the magnitude of effect of biomarkers was similar for men and women, we then performed the analysis adjusting for gender, age, and body mass index (BMI).

Hypothesis 2: imbalance of cartilage synthesis and degradation is predictive of cartilage loss

To test this hypothesis, we grouped biomarkers into those that potentially reflect cartilage synthesis (CPII) and those that reflect cartilage degradation (Col2:3/4 $\mathrm{C}_{\text {long }}[\mathrm{C} 2 \mathrm{C}], \mathrm{Col} 2: 3 /$ $4 \mathrm{C}_{\text {short }}[\mathrm{C} 1,2 \mathrm{C}]$, and Col2CTx). We performed the same analytic approach as above with the predictor variable being a synthesis/degradation marker.

\section{Results}

At baseline, the mean (standard deviation) age was 67 (9) years and $54 \%$ were male. The remainder of the demographic characteristics are presented in Table 1. Seventy-six percent of the subjects had radiographic tibiofemoral OA (Kellgren \& Lawrence [K\&L] grade of greater than or equal to 2) and the remainder had patellofemoral OA. Further descriptive characteristics for the participants are provided according to whether they lost cartilage in any plate during the course of a 30-month follow-up. Compared to those who did lose cartilage at followup, there was an over-representation of women and patients with patellofemoral OA (as opposed to TF OA K\&L grade of greater than or equal to 2) in participants who did not lose cartilage at follow-up. There was no difference in biomarker levels between the two groups at baseline.

Table 1

\section{Baseline characteristics of study population $(n=137)$}

\begin{tabular}{|c|c|c|c|}
\hline & Whole sample & $\begin{array}{l}\text { No cartilage loss in any plate } \\
\text { (at follow-up) }(n=66)\end{array}$ & $\begin{array}{l}\text { Cartilage loss in any plate } \\
\text { (at follow-up) }(n=71)\end{array}$ \\
\hline Age in years, mean $\pm S D$ & $67 \pm 9$ & $66 \pm 9$ & $67 \pm 10$ \\
\hline Males, number (percentage) & $74(54 \%)$ & $38(58 \%)$ & $36(51 \%)$ \\
\hline Body mass index, mean (SD) & $31.43(5.48)$ & $31.03(6.08)$ & $31.75(4.87)$ \\
\hline Percentage with $\mathrm{K} \& \mathrm{~L}$ grade of $\geq 2$ & $73 \%$ & $67 \%$ & $80 \%$ \\
\hline Number of plates with cartilage loss, mean (SD) & $0.99(1.24)$ & $0(0)$ & $1.92(1.09)$ \\
\hline Mean interval in years between baseline and follow-up scan & 2.67 & 2.68 & 2.66 \\
\hline \multicolumn{4}{|l|}{ Levels of biomarkers, mean (SD) } \\
\hline COMP & $12.5(2.9)$ & $11.9(2.9)$ & $13.0(2.9)$ \\
\hline 846 epitope & $269.3(154.2)$ & $273.4(154.6)$ & $265.4(154.8)$ \\
\hline CPII & $1,660.9(598.5)$ & $1,635.9(623.9)$ & $1,684.1(577.3)$ \\
\hline $\mathrm{C} 1,2 \mathrm{C}$ & $0.06(0.03)$ & $0.06(0.03)$ & $0.06(0.03)$ \\
\hline Col2CTX/Cr ratio & $51.77(63.53)$ & $45.82(31.81)$ & $57.62(83.70)$ \\
\hline $\mathrm{C} 2 \mathrm{C}$ & $53.2(22.9)$ & $57.0(28.6)$ & $49.7(15.5)$ \\
\hline
\end{tabular}

C2C, collagenase cleavage of triple-helical type II collagen; Col2CTx, crosslinked peptides from the C-telopeptide domain of type II collagen; COMP, cartilage oligomeric matrix protein; CPII, C-propeptide of type II collagen; K\&L, Kellgren \& Lawrence; SD, standard deviation. 
Table 2

Baseline measures of individual standardized cartilage biomarkers and their respective prediction of subsequent cartilage loss on magnetic resonance imaging

\begin{tabular}{lcclcc}
\hline & $\begin{array}{c}\text { Unadjusted OR } \\
(95 \% \mathrm{Cl})\end{array}$ & $R^{2}$ & AUC & $\begin{array}{c}P \text { value } \\
\text { Adjusted ORa } \\
(95 \% \mathrm{Cl})\end{array}$ \\
\hline COMP & $6.09(1.34,27.67)$ & 0.06 & 0.60 & 0.02 & $6.35(1.36-29.65)$ \\
846 epitope & $0.93(0.51,1.71)$ & 0.001 & 0.52 & 0.82 & $0.96(0.52,1.78)$ \\
CPII & $1.19(0.61,2.32)$ & 0.002 & 0.53 & 0.62 & $1.18(0.60,2.32)$ \\
C1,2C & $0.69(0.30,1.59)$ & 0.008 & 0.55 & 0.38 & $0.72(0.31,1.68)$ \\
Col2CTX/Cr ratio & $0.99(0.65,1.52)$ & 0.000 & 0.52 & 0.97 & $0.95(0.61,1.47)$ \\
C2C & $0.39(0.13,1.13)$ & 0.03 & 0.58 & 0.08 & $0.4(0.14,1.17)$
\end{tabular}

aAdjusted for age, gender, and body mass index. AUC, area under the curve; $\mathrm{C} 2 \mathrm{C}$, collagenase cleavage of triple-helical type II collagen; $\mathrm{Cl}$, confidence interval; Col2CTx, crosslinked peptides from the C-telopeptide domain of type Il collagen; COMP, Cartilage Oligomeric Matrix Protein; CPII, C-propeptide of type II collagen; OR, odds ratio.

Table 3

\begin{tabular}{|c|c|c|c|c|c|}
\hline & $\begin{array}{l}\text { Unadjusted OR } \\
\qquad(95 \% \mathrm{Cl})\end{array}$ & $R^{2}$ & AUC & $P$ value & $\begin{array}{l}\text { Adjusted ORa } \\
(95 \% \mathrm{Cl})\end{array}$ \\
\hline CTX/CPII & $0.72(0.03,16.66)$ & 0.001 & 0.52 & 0.84 & $0.53(0.02,13.23)$ \\
\hline C2C/CPII & $<0.001(<0.001,1.14)$ & 0.04 & 0.60 & 0.05 & $0.001(<0.001,1.46)$ \\
\hline $\mathrm{C} 1,2 \mathrm{C} / \mathrm{CPII}$ & $0.24(0.002,39.12)$ & 0.003 & 0.52 & 0.59 & $0.31(0.002,52.18)$ \\
\hline $\mathrm{C} 1,2 \mathrm{C} / \mathrm{C} 2 \mathrm{C}$ & $0.19(0.02,2.12)$ & 0.02 & 0.57 & 0.18 & $0.22(0.02,2.48)$ \\
\hline Col2CTX/Cr ratio/C2C & $1.60(0.31,8.19)$ & 0.003 & 0.52 & 0.57 & $1.37(0.26,7.40)$ \\
\hline
\end{tabular}

aAdjusted for age, gender, and body mass index. AUC, area under the curve; $\mathrm{C} 2 \mathrm{C}$, collagenase cleavage of triple-helical type II collagen; $\mathrm{Cl}$, confidence interval; Col2CTx, Crosslinked peptides from the C-telopeptide domain of type II collagen; CPII, C-propeptide of type II collagen; OR, odds ratio.

The results of the logistic regression for univariate biomarker predictors with the outcome cartilage loss in any plate are presented in Table 2. With the exception of COMP, none of the other biomarkers was a statistically significant predictor of cartilage loss. For COMP, a 1-standard deviation increase in COMP increased the odds of subsequent cartilage loss 6.09 times (95\% confidence interval [Cl] 1.34 to 27.67). The $\mathrm{C}$ (AUC) statistic for the univariate association was 0.60. After the analysis for COMP was adjusted for age, gender, and BMI, the risk for cartilage loss was 6.35 (95\% Cl 1.36 to 29.65$)$. The results of the logistic regression for imbalance of biomarker predictors with the outcome cartilage loss in any plate are presented in Table 3. Whereas C2C/CPII approached significance, none of the ratios tested facilitated prediction of cartilage loss.

\section{Discussion}

Increased COMP levels predict subsequent cartilage loss on MRI, but the association is modest (area under the curve = 0.60). The other biochemical markers of cartilage synthesis, turnover, and degradation do not facilitate prediction of cartilage loss.
Articular cartilage is a multiphasic material with at least two major phases: a fluid phase composed of water and electrolytes and a solid phase composed of chondrocytes together with matrix molecules that include collagen and proteoglycans. The predominant type of collagen is type II, which is found predominantly in cartilage (some also in the vitreous of the eye). It forms the basic fibrillar structure of the extracellular matrix which imparts its tensile strength.

As articular cartilage degenerates in OA, chondrocytes upregulate their biosynthetic activities, including type II collagen, as if to compensate for this damage. Only after secretion, as the molecules reach the extracellular space, are the non-helical domains at the end (the amino-terminal type II and carboxy-terminal type II procollagen propeptides [PIINP and PIICP, respectively]) cleaved from the helical domain.

The C-propeptide content and release from the cartilage are directly correlated with collagen synthesis [24]. In OA, a variant form of type II collagen is produced in which the N-propeptide contains an additional gene product of exon 2 [25]. This is type IIA collagen and it represents a form found in develop- 
ment but apparently not in healthy adult cartilage. The remainder of the collagen is called type IIB and lacks the exon 2 product. By immunoassay, it is possible to detect both type IIA and IIB collagen with the use of the C-propeptide assay [24].

It is hoped that the availability of assays to measure degradative, synthetic, and turnover products of cartilage matrix metabolism in body fluids offers opportunities to try and monitor cartilage turnover in vivo. Type II collagen is degraded by proteolytic enzymes secreted by chondrocytes and synoviocytes. The cleavage of the type II collagen triple helix by collagenases results in the generation of neoepitopes at cleavage sites. Since the initial cleavage that generates the neoepitope is followed by subsequent cleavage of the alpha chain, there is release of the epitope from the tissue [26]. Thus, an increase in these cleavage products can be detected in vivo by immunoassays with antibodies that recognize cleavage epitopes called $\mathrm{COL} 2-3 / 4 \mathrm{C}_{\text {longmono }}$ (also known as $\mathrm{C} 2 \mathrm{C}$ ) and specific for type II collagen [14], and collagenase cleavage epitopes called COL2-3/4 $\mathrm{C}_{\text {short }}$ or $\mathrm{C} 1,2 \mathrm{C}$, which detect cleavages of both type II and I collagens [15], have been generated and are markedly elevated in experimental OA [27]. These epitopes are generated in OA articular cartilage as shown by Billinghurst and colleagues [15]. However, our investigation suggests that they are not detectably elevated in patients at risk of cartilage loss, and thus their role in predicting OA progression in human MRI studies is questionable based on our data. The inability to detect an increase may be related to the number of joints involved in OA (joint load) compared with RA $[19,28]$. Alternatively, it may be because, in serum, we are unable to distinguish increases in pathology from normal turnover.

Some recent work on type II collagen has suggested that assays for type II collagen degradation, when used alone or in combination or with markers of collagen synthesis, can distinguish populations with knee OA which exhibit progression of joint damage from non-progressors [2,29-31]. The ratio of the type II collagen crosslinking C-telopeptide (CTX-II) to the amino propeptide of type IIA collagen [2] or the ratio of two collagenase-generated cleavage epitopes in the helical region (C1,2C to $\mathrm{C} 2 \mathrm{C})$ [3] each can potentially make this distinction. In one of these clinical studies, progression was identified by the increase in type II collagen cleavage products compared to a decrease in the propeptide marker of synthesis [2]. That study had a number of major limitations, including the control subjects having no radiographic evaluation, incomplete evaluation of OA subjects, and only 12 months of follow-up. However, the greater the distinction between increased degradation and decreased synthesis, the more progression was observed. In another unpublished study of progression [3], the relative amount of primary and secondary cleavage correlated with progression. Both $\mathrm{C} 2 \mathrm{C}$ and $\mathrm{C} 1,2 \mathrm{C}$ epitopes contain the cleavage site generated by collagenase. The greater the amount of the shorter epitope $(\mathrm{C} 1,2 \mathrm{C})$ containing the cleavage site, relative to the longer epitope $(\mathrm{C} 2 \mathrm{C})$, the greater the progression. This suggests that there is a difference in proteolysis linked to progression that leads to increased generation of the shorter $\mathrm{C} 1,2 \mathrm{C}$ epitope. Our investigation did not corroborate these findings, potentially due to differences in study design.

In addition to type II collagen, the second main component of the extracellular matrix of articular cartilage is aggrecan, which, like collagen type II, is almost specific to this tissue. Aggrecan is a proteoglycan composed of a core protein to which GAG chains are covalently attached. The compressive stiffness of articular cartilage is a product of the hydration and swelling of aggrecan, embedded as macromolecular aggregates within the collagen fibrillar network. The monoclonal antibody CS 846 prepared for aggrecan reveals the presence of the largest apparently intact molecules that predominate in fetal cartilages but that are almost absent from healthy adult cartilage [32]. In OA, these larger molecules reappear and increase in amount with increased synthesis of this molecule [17] in synovial fluid and serum $[33,34]$. The 846 monoclonal antibody usually recognizes the epitope on the largest molecules and likely signifies the presence of more recently synthesized molecules [17]. We are unaware of previous human clinical studies that have evaluated this promising biomarker and its relation to knee OA progression. Based upon our investigation, its role in predicting progression in knee OA may be limited, although its early increase has been observed in serum in experimental dog OA [35].

Cartilage oligomeric protein is a pentameric protein of the thrombospondin family which can bind type I, II, and IX collagens [36]. It is synthesized by chondrocytes, synovial cells, and other cells of the skeleton. Its synthesis is increased in chondrocytes and in synovial cells when activated by proinflammatory cytokines [37]. Preliminary plain radiographic studies suggested that COMP may be a useful prognostic marker of disease progression in knee [4-6] and hip [7] OA, and longitudinal analysis of COMP may predict episodic or phasic progression of OA [38]. We were able to corroborate these findings in a knee MRI study, suggesting that this marker may be a useful means of identifying progressors, albeit the estimate was modest.

Some further limitations of this work, some of which are generic to the application of biomarkers, warrant mentioning. Age-related increases are commonly seen in biochemical markers and these may produce variation in both biomarker levels and cartilage loss [39]. Efforts were made to adjust for age in analyses. The BOKS study assessed the local structural changes in the knees only and in only one knee (not both). It may be that other studies that investigate the total body burden of $O A$, including other joint areas, may be able to detect an association in patients with symptomatic OA. Another potential explanation for our null findings in patients with symptomatic OA is that we have insufficient power. It is further pos- 
sible that other biomarkers that we did not measure could have a relation to MRI cartilage loss such as matrix metalloproteinase-3, high sensitive $\mathrm{C}$-reactive protein, hyaluronan, CTX-I, keratan sulphate, matrillin-1, and cartilage intermediate layer protein. In addition, longitudinal analyses of biomarker change would be interesting to explore.

Another potential explanation for the lack of association found relates to limitations with the endpoint, namely cartilage loss on MRI. This was measured semiquantitatively with inherent potential observer bias and possible measurement error. Nonetheless, a number of studies have found plausible biologic associations with cartilage loss on MRI in this dataset, including relations to alignment [40], bone marrow lesions [41], and meniscal abnormalities [42]. Thus, any lack of ability to detect a strong association between biomarkers and MRI is unlikely to be a result of limitations in the MRI variable.

\section{Conclusion}

With the exception of COMP, if changes in cartilage turnover in patients with symptomatic knee OA are associated with cartilage loss, they do not appear to affect systemic biomarker levels. Where there are other markers such as alignment and bone marrow lesions that are potent predictors of progression, we would not advocate one-time measurement of biochemical markers to predict MRI progression in patients with symptomatic knee OA, with the possible exception of COMP.

\section{Competing interests}

The authors declare that they have no competing interests.

\section{Authors' contributions}

$\mathrm{DH}$ conceived of the study, participated in its design and coordination, and drafted the manuscript. JL and $M L$ carried out the statistical analyses. JD carried out the assays. AG and DG read and interpreted the MRIs. DB, MN, RP, DE, and DF participated in the design of the study. All authors read and approved the final manuscript.

\section{Acknowledgements}

The authors thank the participants and staff of the Boston Osteoarthritis Knee Study.

This study was supported by NIH UO1 AR50900 (Osteoarthritis Biomarkers Network), AR47785, and AG18393. The study sponsor was not involved in the study design; in the collection, analysis, and interpretation of data; in the writing of the report; or in the decision to submit the paper for publication.

\section{References}

1. Poole AR: Can serum biomarker assays measure the progression of cartilage degeneration in osteoarthritis? Arthritis Rheum 2002, 46:2549-2552.

2. Garnero P, Ayral X, Rousseau JC, Christgau S, Sandell LJ, Dougados M, Delmas PD: Uncoupling of type II collagen synthesis and degradation predicts progression of joint damage in patients with knee osteoarthritis. Arthritis Rheum 2002, 46:2613-2624.
3. Cerejo R, Poole A, lonescu M, Lobanok T, Song J, Cahue S: The association between cartilage activity measured in serum and progression of knee osteoarthritis in patients with and without evidence of generalized disease. Arthritis Rheum 2002, 46:S144.

4. Petersson IF, Boegard T, Svensson B, Heinegård D, Saxne T: Changes in cartilage and bone metabolism identified by serum markers in early osteoarthritis of the knee joint. $\mathrm{Br} J$ Rheumatol 1998, 37:46-50.

5. Sharif M, Saxne T, Shepstone L, Kirwan JR, Elson CJ, Heinegård D, Dieppe PA: Relationship between serum cartilage oligomeric matrix protein levels and disease progression in osteoarthritis of the knee joint. Br J Rheumatol 1995, 34:306-310.

6. Vilim V, Olejarova M, Machacek S, Gatterova J, Kraus VB, Pavelka $\mathrm{K}$ : Serum levels of cartilage oligomeric matrix protein (COMP) correlate with radiographic progression of knee osteoarthritis. Osteoarthritis Cartilage 2002, 10:707-713.

7. Conrozier T, Saxne T, Fan CS, Mathieu P, Tron AM, Heinegård D, Vignon E: Serum concentrations of cartilage oligomeric matrix protein and bone sialoprotein in hip osteoarthritis: a one year prospective study. Ann Rheum Dis 1998, 57:527-532.

8. Boegård T: Radiography and bone scintigraphy in osteoarthritis of the knee - comparison with MR imaging. Acta Radiol Suppl 1998, 418:7-37.

9. Amin S, LaValley MP, Guermazi A, Grigoryan M, Hunter DJ, Clancy M, Niu J, Gale DR, Felson DT: The relationship between cartilage loss on magnetic resonance imaging and radiographic progression in men and women with knee osteoarthritis. Arthritis Rheum 2005, 52:3152-3159.

10. Felson DT, McLaughlin S, Goggins J, LaValley MP, Gale ME, Totterman S, Li W, Hill C, Gale D: Bone marrow edema and its relation to progression of knee osteoarthritis. Ann Intern Med 2003, 139(5 Pt 1):330-336

11. Chaisson CE, Gale DR, Gale E, Kazis L, Skinner K, Felson DT: Detecting radiographic knee osteoarthritis: what combination of views is optimal? Rheumatology 2000, 39:1218-1221.

12. Buckland-Wright C: Protocols for precise radio-anatomical positioning of the tibiofemoral and patellofemoral compartments of the knee. Osteoarthritis Cartilage 1995, 3(Suppl A):71-80.

13. Peterfy CG, Guermazi A, Zaim S, Tirman PF, Miaux $Y$, White D, Kothari M, Lu Y, Fye K, Zhao S, et al:: Whole-Organ Magnetic Resonance Imaging Score (WORMS) of the knee in osteoarthritis. Osteoarthritis Cartilage 2004, 12:177-190.

14. Kojima T, Mwale F, Yasuda T, Girard C, Poole AR, Laverty S: Early degradation of type IX and type II collagen with the onset of experimental inflammatory arthritis. Arthritis Rheum 2001, 44:120-127.

15. Billinghurst RC, Dahlberg $L$, lonescu $M$, Reiner $A$, Bourne $R$, Rorabeck C, Mitchell P, Hambor J, Diekmann O, Tschesche H, et al.: Enhanced cleavage of type II collagen by collagenases in osteoarthritic articular cartilage. J Clin Invest 1997, 99:1534-1545.

16. Månsson $B$, Carey $D$, Alini $M$, lonescu $M$, Rosenberg LC, Poole $A R$, Heinegård D, Saxne T: Cartilage and bone metabolism in rheumatoid arthritis. Differences between rapid and slow progression of disease identified by serum markers of cartilage metabolism. J Clin Invest 1995, 95:1071-1077.

17. Rizkalla G, Reiner A, Bogoch E, Poole AR: Studies of the articular cartilage proteoglycan aggrecan in health and osteoarthritis. Evidence for molecular heterogeneity and extensive molecular changes in disease. J Clin Invest 1992, 90:2268-2277.

18. Robion FC, Doizé B, Bouré L, Marcoux M, lonescu M, Reiner A Poole AR, Laverty S: Use of synovial fluid markers of cartilage synthesis and turnover to study effects of repeated intra-articular administration of methylprednisolone acetate on articular cartilage in vivo. J Orthop Res 2001, 19:250-258.

19. Verstappen SM, Poole AR, lonescu M, King LE, Abrahamowicz M, Hofman DM, Bijlsma JW, Lafeber FP, Utrecht Rheumatoid Arthritis Cohort Study group (SRU): Radiographic joint damage in rheumatoid arthritis is associated with differences in cartilage turnover and can be predicted by serum biomarkers: an evaluation from 1 to 4 years after diagnosis. Arthritis Res Ther 2006, 8:R31.

20. Mazzuca SA, Poole AR, Brandt KD, Katz BP, Lane KA, Lobanok T: Associations between joint space narrowing and molecular 
markers of collagen and proteoglycan turnover in patients with knee osteoarthritis. J Rheumato/ 2006, 33:1147-1151.

21. Atley L, Shao P, Ochs V, Shaffer K, Eyre D: Matrix metaloproteinase-mediated release of immunoreactive telopeptides from cartilage type II collagen. Trans Orthop Res Soc 1998, 23:850.

22. Lohmander LS, Atley LM, Pietka TA, Eyre DR: The release of crosslinked peptides from type II collagen into human synovial fluid is increased soon after joint injury and in osteoarthritis. Arthritis Rheum 2003, 48:3130-3139.

23. Saxne T, Heinegård D: Cartilage oligomeric matrix protein: a novel marker of cartilage turnover detectable in synovial fluid and blood. Br J Rheumatol 1992, 31:583-591. [Erratum in $\mathrm{Br} J$ Rheumatol 1993, 32:247].

24. Nelson F, Dahlberg L, Laverty S, Reiner A, Pidoux I, lonescu M, Fraser GL, Brooks E, Tanzer M, Rosenberg LC, et al:: Evidence for altered synthesis of type II collagen in patients with osteoarthritis. J Clin Invest 1998, 102:2115-2125.

25. Aigner T, Zhu Y, Chansky HH, Matsen FA III, Maloney WJ, Sandell LJ: Reexpression of type IIA procollagen by adult articular chondrocytes in osteoarthritic cartilage. Arthritis Rheum 1999, 42:1443-1450.

26. Dahlberg L, Billinghurst RC, Manner $P$, Nelson F, Webb G, lonescu M, Reiner A, Tanzer M, Zukor D, Chen J, et al:: Selective enhancement of collagenase-mediated cleavage of resident type II collagen in cultured osteoarthritic cartilage and arrest with a synthetic inhibitor that spares collagenase 1 (matrix metalloproteinase 1). Arthritis Rheum 2000, 43:673-682.

27. Chu $\mathrm{Q}$, Lopez $M$, Hayashi $K$, lonescu M, Billinghurst RC, Johnson KA, Poole AR, Markel MD: Elevation of a collagenase generated type II collagen neoepitope and proteoglycan epitopes in synovial fluid following induction of joint instability in the dog. Osteoarthritis Cartilage 2002, 10:662-669.

28. Poole AR, lonescu M, Fitzcharles MA, Billinghurst RC: The assessment of cartilage degradation in vivo: development of an immunoassay for the measurement in body fluids of type II collagen cleaved by collagenases. J Immunol Methods 2004, 294:145-153.

29. Christgau S, Garnero P, Fledelius C, Moniz C, Ensig M, Gineyts E, Rosenquist $C$, Qvist P: Collagen type II C-telopeptide fragments as an index of cartilage degradation. Bone 2001, 29:209-215.

30. Downs JT, Lane CL, Nestor NB, McLellan TJ, Kelly MA, Karam GA, Mezes PS, Pelletier JP, Otterness IG: Analysis of collagenasecleavage of type II collagen using a neoepitope ELISA. J Immunol Methods 2001, 247:25-34.

31. Charni N, Juillet F, Garnero P, Charni N, Juillet F, Garnero P: Urinary type II collagen helical peptide (HELIX-II) as a new biochemical marker of cartilage degradation in patients with osteoarthritis and rheumatoid arthritis. Arthritis Rheum 2005, 52:1081-1090.

32. Glant TT, Mikecz K, Roughley PJ, Buzás E, Poole AR: Age-related changes in protein-related epitopes of human articular-cartilage proteoglycans. Biochem J 1986, 236:71-75.

33. Lohmander LS, lonescu M, Jugessur $H$, Poole AR: Changes in joint cartilage aggrecan after knee injury and in osteoarthritis. Arthritis Rheum 1999, 42:534-544.

34. Poole AR, lonescu M, Swan A, Dieppe PA: Changes in cartilage metabolism in arthritis are reflected by altered serum and synovial fluid levels of the cartilage proteoglycan aggrecan. Implications for pathogenesis. J Clin Invest 1994, 94:25-33.

35. Matyas JR, Atley L, lonescu M, Eyre DR, Poole AR: Analysis of cartilage biomarkers in the early phases of canine experimental osteoarthritis. Arthritis Rheum 2004, 50:543-552.

36. Clark AG, Jordan JM, Vilim V, Renner JB, Dragomir AD, Luta G, Kraus VB: Serum cartilage oligomeric matrix protein reflects osteoarthritis presence and severity: the Johnston County Osteoarthritis Project. Arthritis Rheum 1999, 42:2356-2364

37. Recklies $A D$, Baillargeon $L$, White C: Regulation of cartilage oligomeric matrix protein synthesis in human synovial cells and articular chondrocytes. Arthritis Rheum 1998, 41:997-1006.

38. Sharif M, Kirwan JR, Elson CJ, Granell R, Clarke S: Suggestion of nonlinear or phasic progression of knee osteoarthritis based on measurements of serum cartilage oligomeric matrix protein levels over five years. Arthritis Rheum 2004, 50:2479-2488

39. Poole AR, Witter J, Roberts N, Piccolo F, Brandt R, Paquin J, Baron $M$ : Inflammation and cartilage metabolism in rheumatoid arthritis. Studies of the blood markers hyaluronic acid, orosomucoid, and keratan sulfate. Arthritis Rheum 1990, 33:790-799.

40. Hunter DJ, Zhang Y, Niu J, Tu X, Amin S, Goggins J, Lavalley M, Guermazi A, Gale D, Felson DT: Structural factors associated with malalignment in knee osteoarthritis: the Boston osteoarthritis knee study. J Rheumatol 2005, 32:2192-2199.

41. Hunter D, Zhang Y, Niu J, Goggins J, Amin S, LaValley M, Guermazi A, Genant $H$, Gale D, Felson DT: Increase in bone marrow lesions is associated with cartilage loss: a longitudinal MRI study in knee osteoarthritis. Arthritis Rheum 2006, 54:1529-1535.

42. Hunter DJ, Zhang YQ, Niu JB, Tu X, Amin S, Clancy M, Guermazi A, Grigorian M, Gale D, Felson DT: The association of meniscal pathologic changes with cartilage loss in symptomatic knee osteoarthritis. Arthritis Rheum 2006, 54:795-801. 